\title{
THE EQUIVALENCE OF TRIPLES OF BILINEAR FORMS*
}

\author{
BY O. E. BROWN
}

1. Introduction. If, in a bilinear form with matrix $\dagger A$, we subject the $x$ 's to a linear transformation with the matrix $C$ and the $y$ 's to a linear transformation with the matrix $B$, we obtain a new bilinear form with the matrix $C^{\prime} A B$, where $C^{\prime}$ is the transpose of $C$. Then two bilinear forms are equivalent if and only if their matrices are equivalent. In this paper we direct our attention entirely to the equivalence of matrices.

It is well known that two $m \times m$ matrices are equivalent if and only if they have the same rank. From classical theory we have also the theorem that two pairs of $m \times m$ matrices are equivalent in the sense of Weierstrass if and only if they have the same invariant factors. We are interested in the extension of the problem to the equivalence of triples of matrices.

2. The General Problem. Given two triples of $m \times m$ matrices

$$
\left(a_{1}, a_{2}, a_{3}\right),\left(\alpha_{1}, \alpha_{2}, \alpha_{3}\right),
$$

to find necessary and sufficient conditions that there exist two nonsingular matrices $P$ and $Q$ such that

$$
P a_{1} Q=\alpha_{1}, P a_{2} Q=\alpha_{2}, P a_{3} Q=\alpha_{3} .
$$

The problem was studied by Sherwood $\ddagger$ who found certain invariants for triples of orders two and three, which, as he says, are not sufficient to characterize all such triples.

It can be easily shown that in the general problem we are at

* Presented to the Society, March 30, 1929.

$\dagger$ It is understood throughout this paper that the elements of all matrices are in the field of all complex numbers.

$\ddagger$ G. E. F. Sherwood, Equivalence of triples of bilinear forms, University of Chicago Doctor's thesis (1922). Mention is made by Sherwood of two papers by S. Kantor, Theorie der Äquivalenz linear $\infty^{\lambda}$ Schaaren bilinear Formen, Sitzungsberichte der Akademie, München, vol. 27 (1897), and Theorie der Elementartheiler höherer Stufen, Monatshefte für Mathematik und Physik, vol. 11 (1900). 
liberty to replace each matrix in one of the triples by a linear combination of the three matrices provided the three linear combinations taken are linearly independent and provided we make the same change in the other triple. If the net $l a_{1}+m a_{2}$ $+n a_{3}$ contains a non-singular matrix it may be reduced to the identity by a preliminary transformation upon the triple. Let us, then, impose the condition that the net contain a non-singular matrix.

3. The Restricted Problem. Given two triples of $m \times m$ matrices

$$
(I ., a, b),(I, \alpha, \beta),
$$

to find necessary and sufficient conditions that there exist a nonsingular matrix $P$ such that

$$
P a P^{-1}=\alpha, P b P^{-1}=\beta .
$$

We shall say that $M$ is a transformation changing $a$ to $\alpha$ if $M$ is a non-singular matrix such that $M a M^{-1}=\alpha$. Furthermore, if such an $M$ exists, $a$ and $\alpha$ will be called similar. In the sequel we shall have use for the following lemma.

LEMMA. If $a, \mu$, and $\alpha$ are three mutually similar matrices and if $P, \Pi$ and $T$ are transformations changing a to $\mu, \alpha$ to $\mu$ and a to $\alpha$, respectively, then we can write

$$
T=\Pi^{-1} G P,
$$

where $G$ is a transformation leaving $\mu$ unaltered.

The hypotheses of the lemma take the form $P a P^{-1}=\mu$, $\Pi \alpha \Pi^{-1}=\mu, T a T^{-1}=\alpha$. It is obviously true that

$$
T=\Pi^{-1} \Pi T P^{-1} P
$$

and by use of the last of the three given relations we obtain

$$
\Pi^{-1} \Pi T P^{-1} P a P^{-1} P T^{-1} \Pi^{-1} \Pi=\alpha .
$$

If we multiply on the left by $\Pi$ and on the right by $\Pi^{-1}$ and use the other two given relations we obtain

$$
\Pi T P^{-1} \mu P T^{-1} \Pi^{-1}=\mu .
$$

Then $\Pi T P^{-1}$ leaves $\mu$ unaltered and may be identified with $G$, which establishes the lemma. 
4. Necessary Conditions. Let us suppose that the two triples $(I, a, b),(I, \alpha, \beta)$ are equivalent and that $M$ is the transformation such that

$$
M a M^{-1}=\alpha, M b M^{-1}=\beta .
$$

Then $a$ and $\alpha$ must be similar to a canonical form $\mu$ while $b$ and $\beta$ must be similar to a canonical form $\nu, \mu$ and $\nu$ being determined by the invariant factors of the characteristic matrices $(a-\lambda I)$ and $(b-\lambda I)$. Now let $P, L, \Pi$ and $\Lambda$ be transformations such that

$$
L a L^{-1}=\mu, \quad P b P^{-1}=\nu, \quad \Lambda \alpha \Lambda^{-1}=\mu, \quad \Pi \beta \Pi^{-1}=\nu .
$$

By the preceding lemma we have

$$
M=\Lambda^{-1} G^{-1} L=\Pi^{-1} H^{-1} P,
$$

where $G$ leaves $\mu$ unaltered while $H$ leaves $\nu$ unaltered. If we multiply on the right by $L^{-1}$ and on the left by $H \Pi$ we have

$$
P L^{-1}=H \Pi \Lambda^{-1} G^{-1} \text {. }
$$

5. Sufficiency Conditions. By reversing the steps in the above argument we see that if this equation is satisfied the triples are equivalent.

6. Notation. Let us call $P L^{-1}$ and $\Pi \Lambda^{-1}$ transpositions of the first and second triples, respectively. The transformation $H \Pi$ changes $\beta$ to $\nu$ as required of $\Pi$, while $G \Lambda$ changes $\alpha$ to $\mu$ as required of $\Lambda$. Hence $H \Pi \Lambda^{-1} G^{-1}$ is also a transposition of the second triple. If, under the above circumstances, there exists a transformation $G$ leaving $\mu$ unaltered and a transformation $H$ leaving $\nu$ unaltered such that $H \Pi \Lambda^{-1} G^{-1}=P L^{-1}$, let us say that the transpositions $P L^{-1}$ and $\Pi \Lambda^{-1}$ are cognate. In this nomenclature we may state our theorem.

Theorem. Two triples of $m \times m$ matrices $(I, a, b)$ and $(I, \alpha, \beta)$ with a similar to $\alpha$ and $b$ similar to $\beta$ are equivalent if and only if their tranpositions are cognate.

One may note that if $\mu$ and $\nu$ are construed as in the classical canonical form, and if the characteristic equations $a-\lambda I=0$ and $b-\lambda I=0$ have no multiple or zero roots, then $\mu$ and $\nu$ are diagonal matrices and so, therefore, $G$ and $H$ are also. In this case as in many others the relation cognate is easily recognized.

Case School of Applied Science 